\title{
Otimização no planejamento da operação de bombas hidráulicas em sistemas de abastecimento de água para redução dos custos com energia elétrica
}

\section{Optimization in planning the operation of hydraulic pumps in water supply systems to reduce costs with electricity}

Data de entrada: 06/05/2020

Data de aprovação: $20 / 08 / 2020$

Letícia Maria Miquelin ${ }^{1}$ Isabela Garcia Parras | Ana Raquel Faccioli | Edilaine Martins Soler*

DOI: https://doi.org/10.36659/dae.2022.012

ORCID ID

Miquelin LM (D) https://orcid.org/0000-0003-1539-843X

Parras IG (D) https://orcid.org/0000-0001-5042-4352
Faccioli AR (D) https://orcid.org/0000-0001-9868-5068

Soler EM (D) https://orcid.org/0000-0002-7615-5768

\section{Resumo}

Diante da crescente demanda por água e energia, faz-se cada vez mais necessário o uso racional e otimizado desses recursos. As despesas com energia elétrica em sistemas de abastecimento representam uma parcela alta de seus custos, e grande parte do consumo de energia deve-se à operação das bombas. Como a tarifa de energia elétrica é diferenciada de acordo com as horas do dia, pesquisas sobre o planejamento da operação das bombas hidráulicas visando à redução dos custos com energia elétrica em tais sistemas vêm sendo feitas há décadas. Devido à dimensão desses sistemas, esse planejamento é uma tarefa complexa. Assim, modelos de otimização e métodos de solução para o planejamento operacional das bombas hidráulicas em sistemas de abastecimento de água têm sido propostos na literatura de forma a viabilizar esse planejamento.

Palavras-chave: Otimização. Bombas Hidráulicas. Sistemas de Abastecimento de Água. Energia Elétrica.

\section{Abstract}

Due to the increased demand for water and energy, the rational and optimized use of these resources is necessary. Expenses with electricity in supply systems represent a high portion of its costs, and a large part of energy consumption is due to the operation of the pumps. As the electricity tariff is differentiated according to the hours of the day, research on the planning of the operation of hydraulic pumps aiming at the reduction of electricity costs in such systems has been carried out in the last decades. Due to the size of the systems, this planning is a complex task. Thus, optimization models and solution methods for the operational planning of hydraulic pumps in water supply systems have been proposed in the literature in order to make this planning feasible.

Keywords: Optimization. Hydraulic Pumps. Water Supply Systems. Electricity.

\footnotetext{
${ }^{1}$ Universidade Estadual Paulista (Unesp), Programa de Pós-Graduação em Engenharia Elétrica- Bauru - São Paulo - Brasil

2 Universidade Estadual Paulista (Unesp), Faculdade de Ciências- Bauru - São Paulo - Brasil

* Autora correspondente: edilaine.soleraunesp.br.
} 


\section{INTRODUÇÃO}

A energia elétrica é indispensável na operação dos sistemas de abastecimento de água, os quais visam obter cada vez mais eficiência na prestação de seus serviços. De acordo com Coelho e Andrade-Campos (2014), os sistemas de abastecimento de água em todo o mundo representam uma parte significativa do consumo global de energia elétrica. Segundo o Diagnóstico dos Serviços de Água e Esgoto, realizado pelo Sistema Nacional de Informações sobre Saneamento (SNIS), há uma tendência crescente de consumo energético no setor, que geralmente acompanha os aumentos nos índices de consumo per capita de água (SNIS, 2019). De La Vega e Alem (2015) estimam que, no Brasil, o setor industrial seja responsável por $48 \%$ do total de energia elétrica consumida no país, onde, desse total, 7\% são consumidos pelos sistemas de distribuição de água.

Segundo Tsutiya (2001) e Van Zyl, Savic e Walters (2004), os custos com energia elétrica ocasionados pelos sistemas de bombeamentos constituem a maior despesa para as empresas de distribuição de água em todo o mundo. Filho e Alcalde (2004) ressaltam que o Brasil é o segundo país com tarifas de energia elétrica mais caras, e o sétimo se não forem incluídos os impostos. Segundo o SNIS (2019), no ano de 2015 houve um aumento de quase 50\% em relação a 2014 nos gastos com energia elétrica, no qual a principal justificativa foram os reajustes tarifários e o sistema de bandeiras tarifárias que entraram em vigor no Brasil. Desse modo, no Brasil e no mundo, é de grande importância que essas empresas realizem um planejamento das operações das bombas hidráulicas para que possam diminuir seus custos com energia elétrica, sem que haja prejuízo no abastecimento de água à população.

Os períodos de ponta (períodos de pico) são horários diários em que ocorre um pico na demanda por energia elétrica. Para evitar uma sobrecarga na rede elétrica, as concessionárias de energia de todo o mundo, visando incentivar o consumo de energia elétrica fora desses períodos, têm adotado a tarifa de energia elétrica dinâmica. $\mathrm{Na}$ tarifação dinâmica, o preço da energia elétrica é maior nos períodos de ponta.

Como as operações de bombeamento são responsáveis por grande parte dos gastos com energia elétrica em sistemas de abastecimento, um planejamento destas, a fim de evitar o seu funcionamento nos horários de ponta, pode representar economias significativas para as empresas de abastecimento. Destaca-se que a implementação de tais ações não requer qualquer investimento por parte das empresas de saneamento em infraestrutura, pois trata-se da utilização de uma programação adequada para o funcionamento das bombas de modo que as demandas sejam atendidas com o menor custo com energia elétrica.

Devido à dimensão dos sistemas de abastecimento, essa tarefa é muito complexa. Para solucionar tal problema, modelos de otimização e métodos de solução têm sido propostos nas últimas décadas para o problema de planejamento das operações de bombeamento (pump scheduling problem). Modelos de otimização têm sido uma ferramenta muito utilizada tanto no planejamento operacional dos sistemas de abastecimento como no desenvolvimento de projetos.

Um modelo de otimização pode ser representado de forma geral por:

Minimizar/Maximizar $\mathrm{f}(\mathrm{x})$

Sujeito a:

$g(x) \leq 0$

$h(x)=0$

$x \in S$ 
em que $\quad x \in \mathbb{R}^{n}, \quad f: \mathbb{R}^{n} \rightarrow \mathbb{R}$, $g: \mathbb{R}^{n} \rightarrow \mathbb{R}^{m}, h: \mathbb{R}^{n} \rightarrow \mathbb{R}^{p}$ e $S \subset \mathbb{R}^{n}$. O vetor $x$ representa as variáveis de decisão do problema. Na Eq. 1, $f(x)$ representa os critérios a serem otimizados (função objetivo); as Eq. 2 e 3, $g(x) \leq 0$ e $h(x)=0$, representam as restrições, e, na Eq. 4, $S \subset \mathbb{R}^{n}$ representa o domínio das variáveis. Nos problemas de planejamento das operações de bombeamento, usualmente a função $f(x)$ representa o custo com o consumo de energia elétrica nas operações de bombeamento e outros custos relacionados à tarifação energética e ao desgaste dos equipamentos.

Este artigo tem como objetivo apresentar uma revisão crítica da literatura sobre a otimização da operação das bombas hidráulicas em sistemas de abastecimento de água visando à redução dos custos com energia elétrica. $O$ artigo está organizado como segue: na seção 2 é apresentada a estrutura tarifária de energia elétrica no Brasil, assim como é realizada a cobrança das empresas responsáveis pelo abastecimento de água; na seção 3 são apresentados dados sobre a literatura acerca do tema em questão; na seção 4 encontra-se um levantamento histórico sobre as principais contribuições na literatura sobre o otimização do planejamento da operação das bombas hidráulicas, e na seção 5 são apresentadas as considerações finais.

\section{TARIFAS DE ENERGIA ELÉTRICA}

Os custos com energia nos sistemas de abastecimento são dados em função do consumo real de energia e da variação das tarifas energéticas (VIERA E RAMOS, 2009). As tarifas energéticas de cada país dependem de suas políticas públicas, de suas capacidades de geração, de sua extensão territorial e de seu Produto Interno Bruto. Sobre essas tarifas incidem impostos e encargos não recuperáveis.
Concessionárias de energia elétrica de todo o mundo têm adotado tarifas de consumo de energia elétrica dinâmicas, ou seja, o preço da energia elétrica é variável de acordo com as horas do dia. Na tarifação dinâmica, o preço da energia elétrica é maior nos períodos do dia denominados de períodos de ponta. Além da taxa referente à energia consumida, outras tarifas podem estar associadas ao uso da energia.

No Brasil, a tarifação energética é definida pela Agência Nacional de Energia Elétrica (ANEEL), que também estabelece os critérios de classificação dos consumidores e as formas de cobrança de energia elétrica. Uma unidade consumidora é classificada pela atividade exercida e, para o faturamento, os consumidores são classificados em dois grupos: Grupo A (alta tensão) e Grupo B (baixa tensão).

O faturamento energético dos consumidores classificados como Grupo A ocorre de maneira binômia, sendo cobrados a demanda energética, que corresponde à média da potência elétrica solicitada pelo consumidor à empresa distribuidora, com a energia consumida. Quando o consumo de potência exceder a demanda contratada em mais de $10 \%$, tem-se uma demanda de ultrapassagem, na qual se aplica uma tarifa que pode ser até três vezes mais cara do que a tarifa de demanda contratada. $O$ objetivo é fazer com que o consumidor faça seu planejamento de consumo de acordo com a demanda contratada, evitando-se, assim, que haja uma sobrecarga no sistema. Já o faturamento energético dos consumidores classificados como Grupo B ocorre de maneira monômia, sendo cobrada apenas a energia consumida.

As empresas de saneamento básico são classificadas como consumidores do Grupo A (ANEEL, 2010), com redução de $15 \%$ das Tarifas de Energia e Tarifas de Uso do Sistema de Distribuição, que são taxas estabelecidas pela Aneel para co- 
brir custos com a geração e transporte da energia (linhas de transmissão e distribuição). Segundo o SNIS (2019), o índice de consumo de energia elétrica pelas empresas de distribuição de água é definido pelo consumo total de energia elétrica, dividido pela soma do volume de água produzido e importado.

Para as empresas de saneamento, a estrutura tarifária utilizada é a horo-sazonal, que pode ser definida como azul ou verde. Ambas as estruturas tarifárias são caracterizadas pela aplicação de tarifas diferenciadas para o consumo de energia elétrica de acordo com a hora do dia e com o período do ano, sendo que a tarifa verde possui uma tarifa única para a demanda energética, enquanto a tarifa azul possui tarifas diferenciadas para tal, de acordo com a hora do dia. O horário de ponta compreende um período de três horas contínuas, entre $17 \mathrm{~h}$ e $22 \mathrm{~h}$, dependendo do estado, de segunda-feira a sexta-feira. No Estado de São Paulo, esse intervalo é das $18 \mathrm{~h}$ às $21 \mathrm{~h}$.

A energia elétrica é um recurso indispensável para a operação dos sistemas de abastecimento de água. $O$ uso de energia representa de 5 a $30 \%$ dos custos de operação desses sistemas no mundo, onde a maior parte desse consumo é dada pelo bombeamento de água (CHAE E KANG, 2013). De acordo com o SNIS (2019), os custos decorrentes do uso de energia em sistemas de saneamento devem ser minimizados e ser alvos de um gerenciamento apropriado, por meio do estabelecimento de ações contínuas de redução e controle de energia, que asseguram benefícios. Portanto, o planejamento da operação das bombas hidráulicas em sistemas de abastecimento de água a fim de evitar a operação destas nos horários de ponta, garantindo o atendimento da demanda da população, pode representar economias significativas para as empresas de abastecimento, e, devido à complexidade do problema, este tem sido bastante investigado na literatura utilizando modelos de otimização.

\section{OTIMIZAÇÃO DA OPERAÇÃO DAS BOMBAS HIDRÁULICAS PARA REDUÇÃO DOS CUSTOS COM ENERGIA ELÉTRICA}

De acordo com Likeman (1994) e Brdys (1994), o objetivo da otimização da operação das bombas em sistemas de abastecimento é fornecer um nível aceitável de serviço aos clientes, atendendo às restrições do sistema e à legislação vigente, minimizando os custos operacionais.

Mala-Jetmarova, Sultanova e Savic (2017) constataram que a maioria dos trabalhos relacionados à otimização nos sistemas de abastecimento de água se concentra em duas áreas: na operação ótima das bombas, de modo a minimizar os custos com energia elétrica dos sistemas de abastecimento de água, e na gestão da qualidade da água, de modo a atender a demanda com água de qualidade e em quantidade adequada.

$\mathrm{Na}$ área de operação ótima das bombas podemos destacar, na base de dados Scopus, o tópico "Sistemas de Abastecimento de Água/Bombas/ Programação das bombas", que contém dados sobre trabalhos relacionados ao tema de 2009 a 2018 , e possui um percentual de destaque de $85,587 \%$. Essa porcentagem é obtida por meio de três métricas: o número de citações e visualizações na base no ano de 2018, para artigos publicados em 2018 e 2017, e o CiteScore médio de 2018. Tal porcentagem reflete o grande destaque que o tema vem recebendo nos últimos anos.

Segundo Mala-Jetmarova, Sultanova e Savic (2017), a maioria dos modelos da literatura para otimização da operação das bombas hidráulicas nos sistemas de abastecimento utiliza a técnica do planejamento explícito das bombas, em que as variáveis de decisão são: o status da bomba (ligada/desligada) em cada intervalo de tempo pré-definido dentro do horizonte de planejamento, ou o tempo em que a bomba deve permanecer ligada, ou o período de início e fim da operação da bomba. Além disso, os autores constataram 
que as redes mais utilizadas na literatura para validação das abordagens são a rede hipotética Anytown (WALSKI et al. 1987), e a rede do exemplo 3 do software de simulação Epanet (USEPA, 2013), baseada no sistema de abastecimento de água de Navato, Califórnia, devido à disponibilidade de informações e dados dessas redes.

A Tabela 1 apresenta as principais características dos modelos de otimização, métodos de solução utilizados e as redes utilizadas nos testes numéricos dos 15 artigos mais citados até 20 de abril de 2020 na base de dados Scopus para o tema, em ordem cronológica. Nesta tabela considere: PL-Programação Linear, PLIM-Programação Linear Inteira-Mista, PNL-Programação Não Linear, PNLIM-Programação Não Linear Inteira-Mista e OM-Otimização Multiobjetivo.

Tabela 1 - Artigos com maior número de citações na base de dados Scopus.

\begin{tabular}{|c|c|c|c|c|}
\hline Referência & Função Objetivo & Restrições & Tipo modelo / Método de solução & Rede testada \\
\hline $\begin{array}{l}\text { Zessler e Shamir } \\
\text { (1989) }\end{array}$ & $\begin{array}{l}\text { Minimizar: custos de } \\
\text { energia da operação das } \\
\text { bombas. }\end{array}$ & $\begin{array}{l}\text { (1) Vazão máxima das bombas; ( } 2 \text { ) } \\
\text { níveis mínimos e máximos de água nos } \\
\text { tanques; (3) níveis inicial e final dos } \\
\text { tanques preestabelecidos. }\end{array}$ & $\begin{array}{c}\text { PNL / Programação dinâmica, utilizando } \\
\text { o método de otimização progressiva } \\
\text { (HOWSON E SANCHO, 1975), combinado } \\
\text { com uma técnica analítica para } \\
\text { otimização local. }\end{array}$ & $\begin{array}{l}\text { (1) Sistema de } \\
\text { distribuição de } \\
\text { água da cidade } \\
\text { de Ein Ziv, Israel, } \\
\text { dividido em } \\
\text { subsistemas. }\end{array}$ \\
\hline $\begin{array}{l}\text { Brion e Mays } \\
\quad(1991)\end{array}$ & $\begin{array}{l}\text { Minimizar: custos de energia } \\
\text { da operação das bombas. }\end{array}$ & $\begin{array}{c}\text { (1) Tempos mínimos e máximos } \\
\text { que uma bomba pode permanecer } \\
\text { ligada em um intervalo de tempo; } \\
\text { (2) valores mínimos e máximos de } \\
\text { pressão para elevar a água nos nós; } \\
\text { (3) níveis mínimos e máximos de água } \\
\text { nos tanques; (4) déficit no volume } \\
\text { dos tanques ao final do horizonte de } \\
\text { planejamento. }\end{array}$ & $\begin{array}{l}\text { PNL / Software KIPipe, usado para resolver } \\
\text { implicitamente as restrições hidráulicas, } \\
\text { integrado com os métodos do Gradiente } \\
\text { Reduzido Generalizado e Função } \\
\text { Lagrangeana Aumentada. }\end{array}$ & $\begin{array}{l}\text { (1) Sistema de } \\
\text { distribuição de } \\
\text { água de parte da } \\
\text { cidade de Austin, } \\
\text { Texas, EUA. }\end{array}$ \\
\hline $\begin{array}{l}\text { Jowitt e } \\
\text { Germanopoulos } \\
\text { (1992) }\end{array}$ & $\begin{array}{l}\text { Minimizar: custos de energia } \\
\text { da operação das bombas, } \\
\text { taxa de consumo de energia } \\
\text { e taxa de demanda. }\end{array}$ & $\begin{array}{l}\text { (1) Horas de bombeamento restritas; } \\
\text { (2) níveis mínimos e máximos de } \\
\text { água nos tanques; (3) níveis inicial e } \\
\text { final de água nos tanques; (4) vazões } \\
\text { mínimas e máximas nos tanques onde } \\
\text { existem válvulas; (5) volume máximo } \\
\text { de captação de água da estação } \\
\text { de bombeamento no horizonte de } \\
\text { planejamento. }\end{array}$ & $\begin{array}{l}\text { PL / Separação entre operação e rede por } \\
\text { meio de suposições. Interação feita por } \\
\text { meio de um modelo de simulação de rede } \\
\text { por período prolongado. As cobranças } \\
\text { máximas de eletricidade são consideradas } \\
\text { por meio de um procedimento iterativo } \\
\text { do problema linear para restrições das } \\
\text { bombas, até que a melhor solução seja } \\
\text { obtida. Por fim, o problema linear é } \\
\text { resolvido utilizando o método } \\
\text { Simplex revisado. }\end{array}$ & $\begin{array}{l}\text { (1) Centro de } \\
\text { telemetria e } \\
\text { telecontrole } \\
\text { Bourne End, na } \\
\text { Divisão Norte da } \\
\text { Thames Water } \\
\text { Authority, em } \\
\text { Buckinghamshire, } \\
\text { Inglaterra. }\end{array}$ \\
\hline $\begin{array}{l}\text { Lanseye } \\
\text { Awumah (1994) }\end{array}$ & $\begin{array}{l}\text { Minimizar: custos de energia } \\
\text { da operação das bombas. }\end{array}$ & $\begin{array}{l}\text { (1) Valores mínimos e máximos de } \\
\text { pressão para elevar a água nos nós; (2) } \\
\text { níveis mínimos e máximos de água nos } \\
\text { tanques; (3) níveis inicial e final de água } \\
\text { nos tanques iguais; (4) número máximo } \\
\text { de liga/desliga das bombas para cada } \\
\text { intervalo de tempo; (5) número máximo } \\
\text { de liga/desliga das bombas para o } \\
\text { horizonte de planejamento. }\end{array}$ & $\begin{array}{l}\text { PNLIM / Abordagem em dois níveis, onde, } \\
\text { primeiro é realizada uma pré-otimização } \\
\text { para geração de dados hidráulicos para o } \\
\text { segundo nível, que é a etapa de otimização, } \\
\text { em que é utilizado programação dinâmica. }\end{array}$ & $\begin{array}{l}\text { (1) Sistema de } \\
\text { distribuição de } \\
\text { água de parte da } \\
\text { cidade de Austin, } \\
\text { Texas, EUA. }\end{array}$ \\
\hline $\begin{array}{l}\text { Yu, Powell e } \\
\text { Sterling (1994) }\end{array}$ & $\begin{array}{l}\text { Minimizar: custos de energia } \\
\text { da operação das bombas. }\end{array}$ & $\begin{array}{l}\text { (1) Conservação de massa nos nós; (2) } \\
\text { vazão máxima e mínima das bombas; } \\
\text { (3) pressão mínima e máxima nos nós; } \\
\text { (4) níveis mínimos e máximos nos } \\
\text { tanques. }\end{array}$ & $\begin{array}{l}\text { PNL / Algoritmo híbrido que utiliza o } \\
\text { método do Gradiente Reduzido j } \\
\text { untamente com uma análise de } \\
\text { sensibilidade não linear. }\end{array}$ & $\begin{array}{l}\text { (1) Rede de médio } \\
\text { porte criada pelos } \\
\text { autores. }\end{array}$ \\
\hline $\begin{array}{l}\text { Mackle, Savic e } \\
\text { Walters (1995) }\end{array}$ & $\begin{array}{l}\text { Minimizar: custos de energia } \\
\text { da operação das bombas } \\
\text { somado a penalidades por } \\
\text { restrições violadas. }\end{array}$ & $\begin{array}{l}\text { (1) Níveis mínimos e máximos de água } \\
\text { nos tanques; (2) demandas dos nós } \\
\text { consumidores a serem atendidas; (3) } \\
\text { déficit no volume dos tanques no final } \\
\text { do horizonte de planejamento. }\end{array}$ & $\begin{array}{l}\text { PNL / Algoritmo genético modificado por } \\
\text { meio da introdução de um procedimento } \\
\text { de classificação, sendo os membros da } \\
\text { população classificados de acordo com } \\
\text { seus custos. }\end{array}$ & $\begin{array}{c}\text { (1) Pequena rede } \\
\text { de distribuição de } \\
\text { água criada pelos } \\
\text { autores. }\end{array}$ \\
\hline $\begin{array}{l}\text { Nitivattananon, } \\
\text { Sadowski e } \\
\text { Quimpo (1996) }\end{array}$ & $\begin{array}{l}\text { Minimizar: custos de energia } \\
\text { da operação das bombas } \\
\text { somado a taxa de demanda. }\end{array}$ & $\begin{array}{l}\text { (1) Limites mínimo e máximo para a } \\
\text { vazão das bombas; (2) Níveis mínimos } \\
\text { e máximos de água nos tanques; (3) } \\
\text { conservação de fluxo; (4) Volume no } \\
\text { final do horizonte de planejamento } \\
\text { igual ao volume no início do horizonte. }\end{array}$ & $\begin{array}{l}\text { PLIM/ O sistema é decomposto em vários } \\
\text { subsistemas e os períodos de planejamento } \\
\text { em períodos operacionais. A otimização } \\
\text { progressiva é aplicada para resolver um } \\
\text { modelo de programação dinâmico. Uma } \\
\text { heurística é utilizada para discretizar as } \\
\text { variáveis relacionadas às bombas. }\end{array}$ & $\begin{array}{l}\text { (1) Sistema de } \\
\text { abastecimento de } \\
\text { água da cidade } \\
\text { de Pittsburgh, } \\
\text { Pensilvânia, EUA. }\end{array}$ \\
\hline
\end{tabular}

continua... 
Tabela 1 - Continuação...

\begin{tabular}{|c|c|c|c|c|}
\hline Referência & Função Objetivo & Restrições & Tipo modelo / Método de solução & Rede testada \\
\hline $\begin{array}{l}\text { Savic, } \\
\text { Walters e } \\
\text { Schwab } \\
(1997)\end{array}$ & $\begin{array}{l}\text { Minimizar: (1) custos de energia } \\
\text { da operação das bombas } \\
\text { somado a penalidades por } \\
\text { restrições violadas; ( } 2 \text { ) número } \\
\text { de acionamentos das bombas. }\end{array}$ & $\begin{array}{l}\text { (1) Níveis mínimos e máximos de água } \\
\text { nos tanques; (2) recuperação do nível } \\
\text { inicial da água no tanque no final do } \\
\text { horizonte de planejamento. }\end{array}$ & $\begin{array}{l}\text { PL / Separação entre operação e rede por } \\
\text { meio de suposições. Interação feita por } \\
\text { meio de um modelo de simulação de rede } \\
\text { por período prolongado. As cobranças } \\
\text { máximas de eletricidade são consideradas } \\
\text { por meio de um procedimento iterativo } \\
\text { do problema linear para restrições das } \\
\text { bombas, até que a melhor solução } \\
\text { seja obtida. Por fim, o problema linear é } \\
\text { resolvido utilizando o método } \\
\text { Simplex revisado. }\end{array}$ & $\begin{array}{l}\text { (1) Centro de } \\
\text { telemetria e } \\
\text { telecontrole } \\
\text { Bourne End, na } \\
\text { Divisão Norte da } \\
\text { Thames Water } \\
\text { Authority, em } \\
\text { Buckinghamshire, } \\
\text { Inglaterra. }\end{array}$ \\
\hline $\begin{array}{l}\text { Cembrano } \\
\text { et al. (2000) }\end{array}$ & $\begin{array}{l}\text { Minimizar: custo de aquisição } \\
\text { da água e custos de energia da } \\
\text { operação das bombas. }\end{array}$ & $\begin{array}{l}\text { (1) Limites operacionais dos níveis dos } \\
\text { tanques; (2) limite de pressão em um } \\
\text { nó de junção; (3) níveis inicial e final nos } \\
\text { tanques são iguais. }\end{array}$ & $\begin{array}{l}\text { PNL / Primeiro é obtido o estado atual } \\
\text { da rede pelo sistema SCADA, em seguida } \\
\text { são previstas demandas futuras usando } \\
\text { o raciocínio indutivo nebuloso. Por fim, a } \\
\text { otimização é executada com o módulo de } \\
\text { controle ideal WATERNET usando o método } \\
\text { de Gradiente Reduzido Generalizado. }\end{array}$ & $\begin{array}{l}\text { (1) Rede reduzida } \\
\text { de Sintra, Portugal. }\end{array}$ \\
\hline $\begin{array}{l}\text { Sakarya e } \\
\text { Mays }(2000)\end{array}$ & $\begin{array}{l}\text { Minimizar: (1) desvios das } \\
\text { concentrações reais dos } \\
\text { constituintes em relação aos } \\
\text { valores desejados e função } \\
\text { penalidade por violar restrições } \\
\text { vinculadas; (2) tempo total de } \\
\text { operação da bomba e função } \\
\text { penalidade por violar restrições } \\
\text { vinculadas; (3) custos de energia } \\
\text { da operação da bomba e função } \\
\text { penalidade por violar restrições } \\
\text { vinculadas. }\end{array}$ & $\begin{array}{l}\text { (1) Limites inferior e superior do tempo } \\
\text { de operação das bombas, limites } \\
\text { inferior e superior da pressão para } \\
\text { elevar a água e limites inferior e superior } \\
\text { dos níveis de água nos tanques; (2) } \\
\text { limites inferior e superior do tempo de } \\
\text { operação da bombas; (3) limites inferior } \\
\text { e superior da pressão para elevar a } \\
\text { água; (4) limites inferior e superior dos } \\
\text { níveis de água nos tanques, (5) limites } \\
\text { inferior e superior nas concentrações } \\
\text { dos constituintes nodais. }\end{array}$ & $\begin{array}{l}\text { PNL / Primeiro é obtido o estado atual } \\
\text { da rede pelo sistema SCADA, em seguida } \\
\text { são previstas demandas futuras usando } \\
\text { o raciocínio indutivo nebuloso. Por fim, a } \\
\text { otimização é executada com o módulo de } \\
\text { controle ideal WATERNET usando o método } \\
\text { de Gradiente Reduzido Generalizado. }\end{array}$ & $\begin{array}{l}\text { (1) Rede reduzida } \\
\text { de Sintra, Portugal. }\end{array}$ \\
\hline $\begin{array}{l}\text { Van Zyl, } \\
\text { Savic e } \\
\text { Walters } \\
(2004)\end{array}$ & $\begin{array}{c}\text { Minimizar: custos de energia } \\
\text { da operação das bombas, } \\
\text { penalidade do déficit nos níveis } \\
\text { dos tanques no final do horizonte } \\
\text { de planejamento e penalidade da } \\
\text { violação do limite de liga/desliga } \\
\text { das bombas. }\end{array}$ & $\begin{array}{l}\text { (1) Níveis mínimos e máximos de água } \\
\text { nos tanques; (2) níveis dos tanques } \\
\text { sem déficit no final do horizonte de } \\
\text { planejamento; (3) limite do número de } \\
\text { liga/desliga da bomba. }\end{array}$ & $\begin{array}{l}\text { PNL / Método híbrido, combinando o } \\
\text { Algoritmo Genético com dois métodos } \\
\text { de busca local, o Hooke e Jeeves e o de } \\
\text { Fibonacci (SCHEWEFEL, 1981). }\end{array}$ & $\begin{array}{l}\text { (1) Rede de } \\
\text { distribuição de } \\
\text { água criada pelos } \\
\text { autores; (2) sistema } \\
\text { de distribuição de } \\
\text { água de Richmond, } \\
\text { Reino Unido. }\end{array}$ \\
\hline $\begin{array}{l}\text { Barán, Von } \\
\text { Lücken } \\
\text { e Sotelo } \\
\text { (2005) }\end{array}$ & $\begin{array}{l}\text { Minimizar: (1) custos de energia } \\
\text { da operação das bombas; } \\
\text { (2) custo de manutenção das } \\
\text { bombas por meio do número } \\
\text { de liga/desliga das bombas; (3) } \\
\text { diferença entre os níveis inicial } \\
\text { e final dos tanques; (4) máximo } \\
\text { pico de potência diário. }\end{array}$ & $\begin{array}{l}\text { (1) Níveis mínimos e máximos de água } \\
\text { nos tanques; (2) pressões mínimas e } \\
\text { máximas na tubulação. }\end{array}$ & $\begin{array}{l}\text { PNL e OM / Algoritmos evolucionários: } \\
\text { Strenght Pareto Evolutionary Algorithm, } \\
\text { Non-Dominated Sorting Genetic Algorithm, } \\
\text { Non-Dominated Sorting Genetic Algorithm } \\
\text { 2, Controlled Elitist Non-Dominated Sorting } \\
\text { Genetic Algorithm, Niched Pareto Genetic } \\
\text { Algorithm e Multiple Objective Genetic } \\
\text { Algorithm combinados com uma heurística } \\
\text { que lida com a restrições hidráulicas. }\end{array}$ & $\begin{array}{c}\text { (1) Sistema } \\
\text { simplificado da } \\
\text { principal estação } \\
\text { de distribuição de } \\
\text { água de Assunção, } \\
\text { Paraguai. }\end{array}$ \\
\hline $\begin{array}{l}\text { López- } \\
\text { lbañez, } \\
\text { Prasad e } \\
\text { Paechter } \\
\text { (2008) }\end{array}$ & $\begin{array}{l}\text { Minimizar: custos de energia da } \\
\text { operação das bombas. }\end{array}$ & $\begin{array}{l}\text { (1) Níveis mínimos e máximos de água } \\
\text { nos tanques; (2) pressão mínima nos } \\
\text { nós de demanda; (3) déficit no nível } \\
\text { do tanque no final do horizonte de } \\
\text { planejamento; (4) número máximo de } \\
\text { liga/desliga das bombas. }\end{array}$ & $\begin{array}{l}\text { PNL / Restrições e limites hidráulicos } \\
\text { nos níveis do tanque são resolvidos } \\
\text { implicitamente pelo simulador hidráulico } \\
\text { Epanet, e demais restrições são resolvidas } \\
\text { pelo algoritmo Colônia de Formigas. }\end{array}$ & $\begin{array}{l}\text { (1) Rede de Van } \\
\text { Zyl, Savic e Walters } \\
\text { (2004); (2) sistema } \\
\text { de distribuição de } \\
\text { água de Richmond, } \\
\text { Reino Unido. }\end{array}$ \\
\hline $\begin{array}{l}\text { Vieira e } \\
\text { Ramos } \\
(2009)\end{array}$ & $\begin{array}{l}\text { Minimizar: custos com energia } \\
\text { em função do nível dos } \\
\text { reservatórios }\end{array}$ & $\begin{array}{l}\text { (1) Níveis mínimos e máximos de água } \\
\text { nos tanques; (2) variação máxima } \\
\text { do nível do reservatório em cada } \\
\text { período devido às capacidades de } \\
\text { bombeamento; (3) variação do nível de } \\
\text { água permitida nos tanques. }\end{array}$ & $\begin{array}{c}\text { PL / Heurística operacional para o liga/ } \\
\text { desliga das bombas e abre/fecha das } \\
\text { válvulas. As soluções são avaliadas no } \\
\text { software EPANET }\end{array}$ & $\begin{array}{l}\text { (1) Sistema realista } \\
\text { simplificado } \\
\text { proposto pelos } \\
\text { autores. }\end{array}$ \\
\hline $\begin{array}{l}\text { Wang, } \\
\text { Chang } \\
\text { e Chen } \\
(2009)\end{array}$ & $\begin{array}{l}\text { Minimizar: (1) custos de energia } \\
\text { da operação das bombas, } \\
\text { número de acionamentos da } \\
\text { bomba e penalidade da violação } \\
\text { do limite de acionamentos das } \\
\text { bombas; (2) tempo total de } \\
\text { funcionamento das bombas e } \\
\text { penalidade da violação do limite } \\
\text { de acionamentos das bombas. }\end{array}$ & $\begin{array}{l}\text { (1) Nível mínimo de água nos tanques; } \\
\text { (2) nível máximo de água nos tanques. }\end{array}$ & $\begin{array}{c}\text { PNL e OM/ Algoritmo Genético } \\
\text { aprimorado proposto pelos autores, } \\
\text { através de uma busca local baseada no } \\
\text { Algoritmo Genético. }\end{array}$ & $\begin{array}{l}\text { (1) Protótipo } \\
\text { de aplicação } \\
\text { implementado } \\
\text { utilizando Delphi } 7 .\end{array}$ \\
\hline
\end{tabular}




\section{HISTÓRICO}

Métodos para a otimização da operação das bombas hidráulicas em sistemas de abastecimento de água vêm sendo desenvolvidos nas últimas décadas devido à pressão sobre esse ramo das empresas de saneamento no sentido de fornecer aos clientes um suprimento contínuo de água em quantidade necessária e de qualidade, visando ao menor custo possível. Nesta seção são descritas as principais características dos trabalhos publicados a partir do final da década de 80, até o início de 2020.

\subsection{Década de 80}

Zessler e Shamir (1989) propuseram um modelo de otimização não linear com o objetivo de minimizar os custos de energia da operação das bombas que utiliza restrições que estabelecem a vazão máxima das bombas e os volumes mínimos e máximos de água nos tanques, além de seus volumes iniciais e finais. São conhecidas as demandas para um período de 24 horas, assim como as propriedades hidráulicas de todos os componentes do sistema de distribuição de água da cidade de Ein Ziv, Israel, dividido em subsistemas. Tal trabalho tem como importância a modelagem proposta, que pode ser aplicada a redes de abastecimento de água nas quais os subsistemas aparecem em qualquer configuração.

Os autores utilizaram a programação dinâmica, por meio do método de otimização progressiva (HOWSON E SANCHO, 1975), combinado com uma técnica analítica para otimização local, para obter a operação ótima das bombas. Para isso, o algoritmo alterna iterativamente entre os horários do dia, que possuem taxas de cobrança de energia elétrica diferenciados, e os subsistemas da rede analisada. Em seguida, o algoritmo converge para o ideal da solução inicial, sendo viável ou não, uma vez que o ideal global é garantido apenas sob certas condições; caso contrário, um local ideal pode ser alcançado.

Little e McCrodden (1989) realizaram um estudo com o objetivo de obter políticas eficientes de bombeamento de água bruta, considerando as taxas energéticas no momento do uso das bombas, por meio de um modelo de programação linear inteiro misto. Considerou-se também o uso geradores de reserva para evitar o uso comercial de energia nos horários de pico. As restrições do problema incluem a satisfação das demandas de água, os níveis mínimo e máximo do tanque e os tempos mínimo e máximo de funcionamento da bomba.

Testes foram realizados com os dados da estação de tratamento de Northside, na cidade de Raleigh, Carolina do Norte, onde a estação de bombeamento é equipada com três geradores a diesel, originalmente adquiridos para fornecer energia de emergência em caso de interrupção no serviço comercial. Com esse modelo, Little e McCrodden (1989) obtiveram uma economia energética de aproximadamente $29 \%$, se comparada à operação realizada na prática do sistema.

\subsection{Década de 90}

Brion e Mays (1991) utilizam o software KYPipe para lidar com restrições hidráulicas e com os volumes mínimos e máximos de água do tanque. Já os limites relacionados à pressão e o déficit no volume do tanque são convertidos em termos de penalidade usando um método lagrangiano adicionado à função objetivo. No primeiro caso de testes é considerado que a decisão de ligar a bomba só pode ser feita no início de cada intervalo de tempo. No segundo caso, a duração do tempo de operação da bomba é uma variável contínua e pode assumir um valor entre zero e até a duração do intervalo de tempo. 
Para o primeiro caso, os resultados de Brion e Mays (1991) indicam uma redução de 5,2\% nos custos com energia elétrica no sistema de distribuição de água de parte da cidade de Austin, comparado à operação real. Para o segundo caso, o custo com energia elétrica sofre uma redução de 17,3\% em relação ao custo operacional real. Ambas as porcentagens representam uma economia por meio, apenas, do planejamento da operação das bombas hidráulicas, sem haver a necessidade de alterações estruturais na rede.

Em Jowitt e Germanopoulos (1992) é apresentado modelo de programação linear para determinar a programação ótima das bombas em um horizonte de planejamento de 24 horas, onde são considerados os custos de energia unitária e demanda máxima, a qual é determinada por meio de um procedimento iterativo de um problema de programação linear para várias restrições no uso da bomba, até que a melhor solução seja obtida. É descrito o uso de simulação de rede por período estendido na determinação dos parâmetros das equações e restrições lineares da rede, e no estudo da operação de rede otimizada, por meio do método Simplex Revisado.

A aplicação do método é realizada numa rede existente no Reino Unido, mostrando que são possíveis economias de até $7,7 \%$ apenas para as cobranças de energia unitárias. Considerando as cobranças de energia unitárias e as cobranças de demanda máxima, Jowitt e Germanopoulos (1992) obtiveram uma economia correspondente a $15,6 \%$. Um dos fatores para essa redução nos custos é o uso das estações de bombeamento mais eficientes em seus limites de uso máximos. Este trabalho destaca-se pois, segundo os autores, a metodologia utilizada é robusta, porém com baixo tempo computacional, tornandose adequada para otimização em tempo real.

Lansey e Awumah (1994) propõem um modelo no qual a operação da bomba em tempo real é resolvida considerando variações nas demandas de água e nos valores cobrados da energia. Uma abordagem de dois níveis é usada para resolver o problema: uma pré-otimização, para gerar resultados hidráulicos e parâmetros de consumo de energia simplificadas por funções não lineares usando o método do quadrado mínimo; e otimização por programação dinâmica. Os autores obtiveram resultados que geraram uma redução de $9 \%$ nos custos com energia elétrica proveniente do sistema de bombeamento em relação à operação real. A solução encontrada gera um menor número de liga/desliga das bombas, ocasionando um menor gasto com manutenção; além de manter o nível do tanque mais baixo do que na operação real, resultando na economia de água potável.

Em Ormsbee e Lansey (1994) são apresentados e discutidos os requisitos e componentes básicos de um ambiente de controle ótimo para sistemas de bombeamento no abastecimento de água. Os componentes básicos incluem modelos de rede hidráulica, previsão de demanda e controle ideais. É apresentada uma revisão das metodologias de controle ótimo existentes para sistemas de bombeamento de abastecimento de água, as quais são classificadas com base no tipo de sistema em que determinada metodologia pode ser aplicada, tipo de modelo hidráulico, tipo de modelo de demanda, tipo de método de otimização e a natureza da política de controle resultante, explanando as vantagens e desvantagens que cada abordagem possui.

Ormsbee e Lansey (1994) elenca e classifica os vários tipos de modelos e algoritmos que foram desenvolvidos até 1994 para solucionar problemas de controle ótimo relacionados a sistemas de abastecimento de água. As formulações dos modelos são classificadas com base na composição física do sistema, e é apresentada uma avaliação geral dos vários algoritmos. A principal vantagem dos modelos de balanço de mas- 
sa apontada por Ormsbee e Lansey (1994) é que sua resposta pode ser rapidamente determinada, diferentemente dos modelos de simulação, sendo estes adequados para uso em conjunto com estratégias de otimização que exigem muitas simulações. Já nos modelos de regressão são destacadas as vantagens em relação a possibilidade da incorporação de um grau de não linearidade do sistema e a eficiência para avaliar sua resposta. Por outro lado, modelos de regressão possuem informações apenas para uma determinada rede em um determinado período; se a rede ou as demandas mudarem, esse tipo de abordagem fornecerá resultados incorretos. Os modelos de simulação são adaptáveis às mudanças do sistema e às variações de demanda espacial; no entanto, exigem modificações do banco de dados ou das curvas de regressão para levar em consideração as alterações na resposta do sistema, além de exigirem maior tempo computacional.

Yu, Powell e Sterling (1994) propuseram um modelo matemático de otimização não linear para determinar a operação ótima de sistemas de distribuição de água contendo várias fontes e tanques. O problema utiliza dados de previsão de demanda de um período de 24 horas para calcular os níveis iniciais e finais nos tanques, assim como para estabelecer as restrições hidráulicas do sistema. Desenvolveu-se um algoritmo de resolução que utiliza o método do gradiente reduzido com uma análise de sensibilidade, onde o algoritmo utiliza uma solução inicial viável, calculando novas soluções viáveis a cada iteração, melhorando gradualmente dentro de uma região factível, até convergir para um ótimo local.

Os autores ressaltam que a precisão do modelo proposto para a programação da bomba é a mesma que a encontrada em simulações hidráulicas, garantindo a viabilidade da solução, em um bom tempo computacional. Yu, Powell e Sterling (1994) ainda destacam que o modelo não exige nenhuma simplificação da rede de distribuição de água a ser testada.

Em meados dos anos 90, iniciou-se o uso de algoritmos heurísticos e metaheurísticos na otimização da operação dos sistemas de abastecimento de água devido ao seu potencial de resolver modelos de programação não linear não convexos, como visto em Mackle, Savic e Walters (1995), que propuseram um modelo com o objetivo de minimizar os custos com energia da operação das bombas hidráulicas, com restrições que limitam níveis mínimos e máximos de água nos tanques, demandas dos nós consumidores a serem atendidas e o déficit no volume do tanque no final do horizonte de planejamento. Os autores utilizaram um Algoritmo Genético que foi modificado por meio da introdução de um procedimento de classificação, sendo os membros da população classificados de acordo com seus custos. Além disso, os autores apontam uma previsão de um aumento do uso de serviços online pelas redes de abastecimento para ajustar os cronogramas planejados para as bombas com o objetivo de compensar as diferenças entre as previsões e as reais demandas dos centros consumidores.

Também na linha dos métodos heurísticos, para resolver o modelo linear inteiro misto proposto, Nitivattananon, Sadowski e Quimpo (1996) decompõem o sistema de abastecimento utilizado em vários subsistemas e os períodos de planejamento em períodos operacionais. A otimização progressiva é aplicada para resolver um modelo de programação dinâmico e uma heurística é utilizada para discretizar as variáveis relacionadas às bombas.

O trabalho de Savic, Walters e Schwab (1997) consiste em uma extensão do trabalho de Mackle, Savic e Walters (1995), sendo implementado um Algoritmo Genético Híbrido, que inclui uma atribuição progressiva de penalidades por vio- 
lações de restrições e uma introdução de viabilidade de soluções como um objetivo adicional para garantir que não haja soluções inviáveis na população final. Tal trabalho tem como objetivo minimizar os custos de energia da operação das bombas, somado a penalidades por restrições violadas, além de minimizar o número de acionamentos das bombas. Primeiramente, resolve-se o modelo apenas com o primeiro objetivo (mono objetivo), sendo, em seguida, utilizados ambos os objetivos para os testes (multiobjetivo). Restrições estabelecendo os níveis mínimos e máximos de água nos tanques foram consideradas, além da recuperação do nível inicial da água no tanque no final do horizonte de planejamento.

As duas abordagens foram testadas alterando-se a quantidade e o perfil da demanda do consumidor com modificações do nível inicial de água no reservatório. Savic, Walters e Schwab (1997) verificaram que mesmo no caso de mudanças grandes (alteração de $25 \%$ no nível inicial do reservatório), a geração da população inicial com as soluções das execuções anteriores trouxe meIhorias na eficiência (velocidade) e na qualidade das soluções encontradas.

\subsection{Década de 2000}

O trabalho de Cembrano et al. (2000) trata do uso de técnicas ótimas de controle em redes de distribuição de água, no qual os autores desenvolveram uma ferramenta de controle ideal integrada a um sistema de controle supervisório, que pode ser usado por uma grande classe de empresas de serviços de água. Inicialmente foi obtido o estado atual da rede do Controle Supervisório e Aquisição de Dados (SCADA). Em seguida, foram previstas as demandas futuras usando o raciocínio indutivo nebuloso. Por fim, a otimização foi executada com o módulo de controle ideal WATERNET, um projeto utilizado neste trabalho que desenvolveu a disseminação de técnicas e ferramentas avançadas para gerenciamento e planejamento da água, usando o método de gradiente reduzido generalizado. Todo o sistema, incluindo o controle ideal, foi demonstrado em um protótipo da rede da área de Sintra, Portugal. Os resultados mostram uma economia de $18 \%$, resultante, principalmente, do bombeamento de água em períodos em que a energia elétrica é mais barata.

O modelo proposto por Sakarya e Mays (2000) para determinar a operação ótima das bombas considera a qualidade da água por meio de um problema de programação ótima de tempo discreto. Já a metodologia de solução é baseada em programação matemática, resultando, assim, em um problema de programação não linear de larga escala, o qual não pode ser resolvido por meio dos métodos não lineares clássicos. Para isso, por meio da interface do software Epanet e o código de otimização não linear GRG2 é possível obter a solução do problema de otimização.

Para testar a eficácia da metodologia proposta, Sakarya e Mays (2000) consideram um sistema hipotético de distribuição de água. Para as três funções objetivo do modelo foi encontrado um cronograma ideal de bombeamento, considerando a qualidade da água. A metodologia proposta, porém, pode ser impraticável, pois pode resultar em um tempo de bombeamento muito curto durante um intervalo de tempo, fazendo com que as bombas sejam ligadas e desligadas excessivamente, ocasionando o desgaste de tais.

Almeida et al. (2001) propuseram um modelo não linear inteiro misto de otimização multiobjetivo visando minimizar a vazão bombeada e a potência de eixo, considerando características como a conservação da massa nos nós da rede, conservação da energia, altura manométrica das bombas e seu rendimento hidráulico. São utilizados dois algoritmos de programação não linear associados a um algoritmo de programação in- 
teira, obtendo uma redução de $36,15 \%$ no consumo de energia elétrica. Além disso, os autores recomendam o uso de um gerador a óleo diesel durante os períodos de ponta para a minimização dos gastos com energia elétrica.

A comparação entre as políticas operacionais de Almeida et al. (2001) tem por finalidade avaliar o desempenho dos algoritmos diante de duas formulações distintas da função objetivo (linear e não linear) e identificar qual a melhor solução, não só em termos de menor consumo de energia, mas também quanto à viabilidade de aplicação e implementação dos resultados fornecidos pelo modelo.

Mesmo presente em trabalhos anteriores, como em Brion e Mays (1991) e Sakarya e Mays (2000), observa-se o aumento no uso de modelos de otimização associados a simuladores hidráulicos, sendo o mais comum o software Epanet, utilizado por Van Zyl, Savic e Walters (2004), Giacomello, Kapelan e Nicolini (2013), Kurek e Ostfeld (2013), Ghaddar et al. (2015), Bonvin et al. (2017), Vieira et al. (2020), entre outros; de modo a garantir soluções viáveis, tanto em relação ao modelo de otimização como às exigências hidráulicas do sistema.

Em Van Zyl, Savic e Walters (2004) é proposto um modelo que visa determinar ações de controle em um sistema de abastecimento para reduzir os custos com energia elétrica resolvido por meio de um método de solução híbrido, que combina o Algoritmo Genético com métodos de busca local. Essa combinação foi proposta, segundo os autores, devido ao fato de o Algoritmo Genético encontrar a região da solução ótima do problema com eficiência, mas não o ponto ótimo, que é melhor encontrado por meio de métodos de busca direta, que exploram a vizinhança de uma solução para melhorá-la.

Para a rede de Richmond, Van Zyl, Savic e Walters (2004) conseguiram uma redução nos gastos com energia elétrica apenas com o planejamento da operação das bombas em relação ao que é feito na prática. Além disso, uma das melhorias alcançadas pelo trabalho foi a conexão do software Epanet à biblioteca do Algoritmo Genético do centro de sistemas de água da Universidade de Exeter, reduzindo o tempo de execução de 69 horas, obtido anteriormente por Atkinson et al. (2000), para 21 horas.

A importância do trabalho de Van Zyl, Savic e Walters (2004) é, não somente, por seu modelo proposto e método de solução, mas também pela criação de uma rede de abastecimento fictícia amplamente utilizada por autores em trabalhos posteriores, como em López-lbañez, Prasad e Paechter (2008) e Vieira et al. (2020), por conta da facilidade na obtenção dos dados, ajudando na comparação de resultados para comprovação da eficiência dos modelos em relação a redução de custos com energia elétrica em sistemas de abastecimento de água.

Barán, Von Lücken e Sotelo (2005) propõem um modelo multiobjetivo para a operação ótima das bombas hidráulicas nos sistemas de abastecimento de água resolvido por meio de seis algoritmos evolucionários multiobjetivos combinados com uma heurística que lida com a restrições hidráulicas, e seus resultados são comparados usando um conjunto de seis métricas propostas em Van Veldhuizen (1999). Os resultados mostram que o Strength Pareto Evolutionary Algorithm fornece o maior número de soluções ideias para o modelo, seguido pelo Non-Dominated Sorting Genetic Algorithm 2.

López-Ibañez, Prasad e Paechter (2008) propõem um modelo matemático que utiliza restrições que limitam a quantidade de liga/desliga das bombas durante o horizonte de planejamento, permitindo um espaço de busca reduzido, o que diminui o tempo computacional do algoritmo, que é resolvido por meio do Algoritmo Colônia de Formigas. 
Tais restrições acarretaram um gasto menor com a energia elétrica na comparação dos resultados com os de Van Zyl, Savic e Walters (2004).

López-Ibañez, Prasad e Paechter (2008) destacam mais vantagens em seus resultados em comparação aos de Van Zyl, Savic e Walters (2004), onde os horários flexíveis gerados pelo Algoritmo Genético híbrido podem produzir intervalos de tempo curtos entre o liga/desliga da bomba, ocasionando seu desgaste, além de alterações de pressão que podem danificar a rede, gerando um aumento os gastos com manutenção, características que não estão presentes em López-lbañez, Prasad e Paechter (2008), por conta da restrição sobre os acionamentos.

O trabalho de Ostfeld e Tubaltzev (2008) é um exemplo de que é possível otimizar os gastos com energia elétrica relacionados ao bombeamento na fase de construção do sistema de abastecimento. Neste é descrito um modelo matemático de otimização não linear com os objetivos de minimizar os custos de construção da tubulação e bombas, assim como os custos de operação das bombas. $O$ modelo ainda minimiza o custo de construção dos tanques e uma função penalidade por violação de pressão nos nós. Para sua resolução é utilizada a metaheurística Colônia de Formigas, e as soluções encontradas são avaliadas no simulador hidráulico Epanet; comparadas aos resultados obtidos por Maier et al. (2003), o algoritmo proposto encontrou melhores resultados para a rede Anytown (WALSKI et al., 1987).

Verifica-se que trabalhos como os citados são de grande dificuldade, pois, segundo Castelletti, Pianosi e Soncini-Sessa (2008), a complexidade do sistema físico, que é não linear e cercado por incertezas, mistura-se com o contexto socioeconômico em que as decisões sobre os sistemas de abastecimento de água têm de ser tomadas.

Nesta década surgiram os primeiros trabalhos com considerações ambientais nos modelos pro- postos e nas análises realizadas. Vieira e Ramos (2009) propõem a instalação de geradores de energia eólica associados ao sistema de abastecimento e avaliam o impacto da utilização dessa energia renovável no planejamento da operação das bombas hidráulicas.

Wang, Chang e Chen (2009) propõem um método de programação das bombas baseado no Algoritmo Genético, no qual as bombas precisam ser usadas intermitentemente, não apenas para redução de custos, mas também para a proteção do meio ambiente, característica que não é considerada em trabalhos anteriores. Nessa metodologia, é realizada uma busca local binária baseada no Algoritmo Genético para aumentar a força de intensificação, onde se destaca uma codificação cromossômica em número real para atender às necessidades dos problemas reais, já que trabalhos anteriores trouxeram uma codificação de tempo simplificada, de difícil flexibilidade, para aumentar a economia de energia.

O algoritmo proposto foi implementado utilizando Delphi 7, um protótipo de aplicativo. $\mathrm{Na}$ primeira fase de testes foi implementado o Algoritmo Genético aprimorado de Savic, Walters e Schwab (1997) para avaliar o algoritmo proposto pelos autores em relação à qualidade da solução, ou seja, custo de eletricidade ou benefícios ambientais. Em todas as simulações, os resultados de Wang, Chang e Chen (2009) geraram melhores soluções.

$\mathrm{Na}$ segunda fase, dois objetivos são otimizados simultaneamente. $O$ algoritmo evolucionário Strength Pareto Evolutionary Algorithm (SPEA) de Barán, Von Lücken e Sotelo (2005) foi implementado e comparado com o algoritmo proposto. O algoritmo proposto por Wang, Chang e Chen (2009) pôde fornecer soluções ecologicamente corretas e econômicas; no entanto, ao buscar custo de energia menor, não superou o SPEA. Segundo os autores, uma vez que o custo de 
eletricidade é aprimorado, é difícil melhorar os benefícios ambientais, de modo que o Algoritmo Genético aprimorado proposto irá sacrificar os benefícios ambientais até um determinado ponto ao executar teste com dois objetivos. Mesmo assim, o método resolveu o problema de agendamento de bombas com maior eficiência do que os métodos convencionais, pois a codificação cromossômica em número real o torna mais flexível e a busca local binária o força a explorar cada área possível, complementando, assim, a codificação de número real e também produzindo soluções quase ideais.

\subsection{De 2010 a 2020}

Giacomello, Kapelan e Nicolini (2013) propuseram um modelo matemático não linear com o objetivo de minimizar os custos de energia elétrica das bombas hidráulicas por meio do planejamento do funcionamento destas com restrições que incluem pressão mínima nos nós, níveis mínimos e máximos de água nos tanques e recuperação do nível no final do horizonte de planejamento, além de mantê-los constantes. Tal modelo é resolvido por meio de um método de otimização em dois estágios.

Primeiro, o modelo é linearizado e a programação linear é aplicada para encontrar um valor quase ideal de solução. Depois toda a linearização é removida e utiliza-se o Greedy Algorithm, um método de busca local, com o Epanet, para explorar a vizinhança de soluções identificadas, a fim de melhorá-las. Esse procedimento permitiu a Giacomello, Kapelan e Nicolini (2013) obter soluções de maneira computacionalmente eficiente para as duas redes de abastecimento testadas: a rede hipotética Anytown (WALSKI, 1987) e a rede de Richmond, em Londres.

No trabalho de Kurek e Ostfeld (2013) são propostos dois modelos de otimização multiobje- tivo, ambos com os objetivos de minimizar os custos com energia elétrica da operação das bombas hidráulicas e os custos de construção dos tanques, porém diferenciados pelo terceiro objetivo, relacionado à qualidade da água; sendo que o primeiro visa minimizar a concentração de desinfetantes na água e o segundo visa minimizar a idade da água a ser distribuída. Restrições físicas da rede são consideradas, assim como a limitação do déficit no nível do tanque no final do horizonte de planejamento. Além disso, restrições de confiabilidade de armazenamento são apresentadas, garantindo que a quantidade de água armazenada nos tanques em cada período seja igual ou superior à demanda prevista para o próximo período do horizonte de planejamento.

A metodologia de resolução utilizada por Kurek e Ostfeld (2013) foi o Strength Pareto Evolutionary Algorithm com o software hidráulico Epanet. Verifica-se que a operação dos tanques é significativamente diferente para dois problemas de otimização, sendo que, quando considerada a desinfecção pelo uso de cloro há um maior armazenamento de água nos tanques, o que implica num aumento dos custos com energia elétrica, e, quando considerada a idade da água, os níveis dos tanques permanecem quase constantes.

Ghaddar et. al. (2015) propõem um modelo de otimização não linear não convexo com o objetivo de minimizar os custos de operação das bombas hidráulicas com o uso de restrições que estabelecem um limite superior para os fluxos nas tubulações, sendo que a bomba deve estar ligada para que a água flua no tubo correspondente. Os níveis mínimos e máximos de água dos reservatórios são estabelecidos, assim como a não-negatividade para os fluxos nas tubulações. A fim de evitar um desgaste das bombas por acionamentos excessivos, foi determinado o tempo mínimo em que a bomba deve permanecer ligada e desligada, além do número máximo de acionamentos permitidos. 
São usados dois esquemas de preços de eletricidade. O primeiro é um esquema fixo de dia/ noite, e o segundo, um esquema dinâmico com preços alterando a cada 30 minutos. Uma abordagem de decomposição lagrangiana é explorada, sendo o problema decomposto de acordo com os períodos em subproblemas menores. Devido ao relaxamento do problema original, as soluções dos subproblemas podem não ser viáveis para o problema original. 0 simulador hidráulico Epanet foi utilizado para verificar se o planejamento obtido atendia à demanda e para calcular o consumo de energia. $O$ impacto de tal esquema de precificação de eletricidade nos custos operacionais da bomba realizado por Ghaddar et al. (2015) é avaliado, e o preço dinâmico resulta em até $34 \%$ de redução do custo com energia elétrica comparado ao custo obtido pelo esquema fixo dia/noite.

Bonvin et al. (2017) propuseram um modelo de programação não linear inteiro misto com o objetivo de minimizar os custos com energia elétrica por meio de um planejamento de operação das bombas hidráulicas no qual, o modelo, que é não convexo, foi relaxado, descartando-se as não-convexidades, sendo apresentada uma função para converter uma solução desse relaxamento em uma solução viável, com um número limitado de aumento do custo.

O modelo de Bonvin et al. (2017) foi testado para a Rede FRD, um sistema de distribuição de água potável localizada em uma zona rural da França, o que mostra a viabilidade do uso de modelos de otimização para a redução dos custos com energia elétrica não apenas em sistemas urbanos. Testes foram realizados considerando um ano de dados históricos do sistema em questão, que resultaram em benefícios práticos comparados à estratégia interna atualmente utilizada na concessionária, como planos de bombeamento para o dia seguinte gerados em menos de um minuto e com sua viabilidade avaliada no simulador Epanet.
Vieira et al. (2020) apresentam uma formulação matemática que inclui aspectos relacionados ao estado do sistema quando os tanques estão cheios, não considerado em trabalhos anteriores. Também é apresentada uma técnica de linearização que inclui o número de liga/desliga das bombas, resultando em um número menor de variáveis binárias para o problema, e o relaxamento proposto, que diminui o espaço de busca das soluções.

Testes realizados por Vieira et al. (2020) superam os melhores resultados encontrados para a rede de Van Zyl, Savic e Walters (2004) até então, obtendo uma solução 1,62\% menor do que a encontrada por Marchi, Simpson e Lambert (2016); para a rede esqueleto de Richmond, com uma redução de 10,82\% em relação a solução encontrada por Giacomello, Kapelan e Nicolini (2013); e para a rede completa de Richmond, com a solução reduzida em 3,2\% em relação a de López-Ibañez, Prasad e Paechter (2008). Os autores ainda contribuem para trabalhos futuros fornecendo uma nova rede de testes de acordo com os parâmetros e condições tarifárias energéticas brasileiras, obtida a partir do sistema de abastecimento de água de Florianópolis, Santa Catarina, Brasil, que, por meio de testes com o modelo proposto, apresentou uma redução de $16,82 \%$ nos gastos com energia elétrica em relação ao que é realizado na prática.

\section{CONSIDERAÇÕES FINAIS}

O desenvolvimento de modelos e métodos de solução para otimização energética do planejamento da operação das bombas em sistemas de abastecimento tem sido motivado pela aplicação dos resultados em redes de abastecimento reais. Os trabalhos mais antigos propuseram modelos de otimização simplificados, em que muitas características das redes de abastecimento não são abordadas e utilizaram métodos exatos de 
solução para tais modelos. Devido a limitação dos recursos computacionais, estas metodologias usualmente foram testadas em redes reais de pequeno porte.

Na década de 90 surgiram os primeiros trabalhos que utilizaram métodos de solução heurísticos e meta-heurísticos para tais problemas, sendo ainda muito utilizados os métodos de solução exatos. Nesta mesma década também surgiram os primeiros trabalhos que utilizaram softwares de cálculos hidráulicos. Na década de 2000, houve uma predominância na utilização de métodos de solução meta-heurísticos integrados a softwares de simulação hidráulica, sendo o principal software utilizado o EPANET. Formulações que consideram o uso e a geração de energias renováveis pelos sistemas de abastecimento também foram propostas, bem como considerações ambientais foram incluídas nos modelos propostos.

A partir de 2010 as formulações para os modelos desenvolvidos tem considerado uma ampla gama de aspectos reais das rede relacionados as especificidades hidráulicas, o que tem tornado esses modelos mais completos e realistas. Devido à dimensão dos sistemas e a complexidade de tais modelos, novos métodos de solução integrados com o software EPANET têm sido desenvolvidos.

Nas abordagens propostas para otimização energética do planejamento da operação das bombas em sistemas de abastecimento notouse uma mudança de paradigma em função do tempo devido à evolução dos recursos computacionais e dos métodos de otimização.

Trabalhos futuros devem atentar-se às novas características apresentadas pelos sistemas de abastecimento de água. A crescente automação destes, especialmente no que diz respeito ao funcionamento das bombas hidráulicas, traz a necessidade de modelos matemáticos que abordem o uso de novas tecnologias e de seus impactos nas redes de distribuição e no consumo de ener- gia elétrica. Além disso, a crescente demanda por eletricidade, assim como a busca por matrizes energéticas limpas e de baixo custo, traz a necessidade de modelos matemáticos que investiguem as vantagens presentes em investimentos na diversificação de matrizes energéticas em sistemas de abastecimento de água, principalmente no uso e geração de energias renováveis.

\section{AGRADECIMENTOS}

O presente trabalho foi realizado com o apoio da Coordenação de Aperfeiçoamento de Pessoal de Nível Superior - Brasil (CAPES) - Código de Financiamento 001 e do Conselho Nacional de Desenvolvimento Científico e Tecnológico (CNPq) - Processos No: 313495/2017-3 e 134096/2018-5.

\section{CONTRIBUIÇÃO DOS AUTORES}

Levantamento Bibliográfico: Miquelin LM, Parras IG, Faccioli AR, Soler EM; Revisão Crítica da Literatura: Miquelin LM, Parras IG, Faccioli AR; Redação da Primeira Versão: Miquelin LM, Parras IG, Faccioli AR; Redação Final: Soler EM; Revisão: Miquelin LM, Parras IG, Faccioli AR, Soler EM; Supervisão: Soler EM.

\section{REFERÊNCIAS}

ALMEIDA, R. et al. Operação de sistemas urbanos de abastecimento de água com base em modelos de otimização não-lineares. 2001.

ANEEL. Resolução Normativa n. 414. [S.I.], 2010. Disponível em: <http://www2.aneel.gov.br/cedoc/bren2010414.pdf>. Acesso em: 17 ago. 2017.

ATKINSON, R. et al. Genetic algorithm optimisation of level-controlled pumping station operation. Water network modelling for optimal design and management, p. 79-90, 2000.

BAGIROV, A. M. et al. An algorithm for minimization of pumping costs in water distribution systems using a novel approach to pump scheduling. Mathematical and Computer Modelling, v. 57, n. 3-4, p. 873-886, 2013. https://doi.org/10.1016/j. mcm.2012.09.015 
BARÁN, B.; VON LÜCKEN, C.; SOTELO, A. Multi-objective pump scheduling optimisation using evolutionary strategies. Advances in Engineering Software, v. 36, n. 1, p. 39-47, 2005. https://doi. org/10.1016/j.advengsoft.2004.03.012

BONVIN, G. et al. A convex mathematical program for pump scheduling in a class of branched water networks. Applied Energy, v. 185, p. 1702-1711, 2017. https://doi.org/10.1016/j.apenergy.2015.12.090

BRDYS, M. A. Operational control of water systems: structures, algorithms, and applications. Prentice Hall, 1994.

BRION, L. M.; MAYS, L. W. Methodology for optimal operation of pumping stations in water distribution systems. Journal of Hydraulic Engineering, v. 117, n. 11, p. 1551-1569, 1991. https:// doi.org/10.1061/(ASCE)0733-9429(1991)117:11(1551)

CASTELLETTI, A.; PIANOSI, F.; SONCINI-SESSA, R. Integration, participation and optimal control in water resources planning and management. Applied Mathematics and Computation, v. 206, n. 1, p. 21-33, 2008. https://doi.org/10.1016/j.amc.2007.09.069

CEMBRANO, G. et al. Optimal control of a water distribution network in a supervisory control system. Control engineering practice, v. 8, n. 10, p. 1177-1188, 2000. https://doi.org/10.1016/ S0967-0661(00)00058-7

CHAE, K.; KANG, J. Estimating the energy independence of a municipal wastewater treatment plant incorporating green energy resources. Energy Conversion and Management, v. 75, p. 664-672, 2013. https://doi.org/10.1016/j.enconman.2013.08.028

CHASE, D. V.; ORMSBEE, L. E. Optimal pump operation of water distribution systems with multiple storage tanks. In: Water Resources Planning and Management. ASCE, 1989. p. 733-736.

COELHO, B.; ANDRADE-CAMPOS, A. Efficiency achievement in water supply systems-A review. Renewable and Sustainable Energy Reviews, v. 30, p. 59-84, 2014. https://doi.org/10.1016/j. rser.2013.09.010

DE CASTRO, N. et al. As tarifas de energia elétrica no Brasil e em outros países: o porquê das diferenças. Rio de Janeiro: CPFL, 2015.

DE LA VEGA, J.; ALEM, D. Energy Rationalization in Water Supply Networks via Stochastic Programming. IEEE Latin America Transactions, v. 13, n. 8, p. 2742-2756, 2015. https://doi.org/10.1109/ TLA.2015.7332158

FILHO, G. S.; ALCALDE, J. L. Análise de Qualidade de Energia e Eficiência Energética em Sistemas de Água e Saneamento. IV SEREASeminário Hispano Brasileiro sobre Sistemas de Abastecimento Urbano de Água, João Pessoa-Brasil, CD-ROM, 2004. Anais...

GHADDAR, B. et al. A Lagrangian decomposition approach for the pump scheduling problem in water networks. European Journal of Operational Research, v. 241, n. 2, p. 490-501, 2015. https://doi. org/10.1016/j.ejor.2014.08.033
GIACOMELLO, C.; KAPELAN, Z.; NICOLINI, M. Fast hybrid optimization method for effective pump scheduling. Journal of Water Resources Planning and Management, v. 139, n. 2, p. 175-183, 2013. https://doi.org/10.1061/(ASCE)WR.1943-5452.0000239

HOWSON, H. R.; SANCHO, N. G. F. A new algorithm for the solution of multi-state dynamic programming problems. Mathematical programming, v. 8 , n. 1, p. 104-116, 1975. https://doi. org/10.1007/BF01580431

JOWITT, P. W.; GERMANOPOULOS, G. Optimal pump scheduling in water-supply networks. Journal of Water Resources Planning and Management, v. 118, n. 4, p. 406-422, 1992. https://doi. org/10.1061/(ASCE)0733-9496(1992)118:4(406)

KUREK, W.; OSTFELD, A. Multi-objective optimization of water quality, pumps operation, and storage sizing of water distribution systems. Journal of environmental management, v. 115, p. 189197, 2013. https://doi.org/10.1016/j.jenvman.2012.11.030

LANSEY, K. E.; AWUMAH, K. Optimal pump operations considering pump switches. Journal of Water Resources Planning and Management, v. 120, n. 1, p. 17-35, 1994. https://doi.org/10.1061/ (ASCE)0733-9496(1994)120:1(17)

LASDON, L. S., WAREN, A. D. GRG2 User's Guide. Department of General Business, University of Texas at Austin. Austin, Texas, 1986.

LIKEMAN, M. Constraint satisfaction methods in water supply scheduling. In: Integrated computer applications in water supply (vol. 1) methods and procedures for systems simulation and control. 1994. p. 213-225.

LITTLE, K. W.; MCCRODDEN, B. J. Minimization of raw water pumping costs using MILP. Journal of Water Resources Planning and Management, v. 115, n. 4, p. 511-522, 1989. https://doi. org/10.1061/(ASCE)0733-9496(1989)115:4(511)

LÓPEZ-IBÁÑEZ, M.; PRASAD, T. D.; PAECHTER, B. Ant colony optimization for optimal control of pumps in water distribution networks. Journal of water resources planning and management, v. 134, n. 4, p. 337-346, 2008. https://doi.org/10.1061/ (ASCE)0733-9496(2008)134:4(337)

MACKLE, G.; SAVIC, G. A.; WALTERS, Go. A. Application of genetic algorithms to pump scheduling for water supply. In: First international conference on genetic algorithms in engineering systems: innovations and applications. IET, 1995. p. 400-405. Anais... https://doi.org/10.1049/cp:19951082

MAIER, H. R. et al. Ant colony optimization for design of water distribution systems. Journal of water resources planning and management, v. 129 , n. 3, p. 200-209, 2003. https://doi. org/10.1061/(ASCE)0733-9496(2003)129:3(200)

MALA-JETMAROVA, H.; SULTANOVA, N.; SAVIC, D. Lost in optimisation of water distribution systems? A literature review of system operation. Environmental modelling \& software, v. 93, p. 209254, 2017. https://doi.org/10.3390/w10030307 
MARCHI, A.; SIMPSON, A. R.; LAMBERT, M. F. Optimization of pump operation using rule-based controls in EPANET2: New ETTAR toolkit and correction of energy computation. Journal of Water Resources Planning and Management, v. 142, n. 7, p. 04016012 , 2016. https://doi.org/10.1061/(ASCE)WR.1943-5452.0000637

NITIVATTANANON, V.; SADOWSKI, E. C.; QUIMPO, R. G. Optimization of water supply system operation. Journal of Water Resources Planning and Management, v. 122, n. 5, p. 374-384, 1996. https://doi.org/10.1061/(ASCE)0733-9496(1996)122:5(374)

ORMSBEE, L. E.; LANSEY, K. E. Optimal control of water supply pumping systems. Journal of Water Resources Planning and Management, v. 120, n. 2, p. 237-252, 1994. https://doi.org/10.1061/ (ASCE)0733-9496(1994)120:2(237)

OSTFELD, A.; TUBALTZEV, A. Ant colony optimization for least-cost design and operation of pumping water distribution systems. Journal of water resources planning and management, v. 134, n. 2, p. 107-118, 2008. https://doi.org/10.1061/(ASCE)07339496(2008)134:2(107)

ROSSMAN, L. A. EPANET users guide. US Enviromental Protecton Agency, Cincinnati, 1994.

SAKARYA, A. B. A.; MAYS, L. W. Optimal operation of water distribution pumps considering water quality. Journal of Water Resources Planning and Management, v. 126, n. 4, p. 210-220, 2000. https://doi.org/10.1061/(ASCE)07339496(2000)126:4(210)

SAVIC, D. A.; WALTERS, G. A.; SCHWAB, Martin. Multiobjective genetic algorithms for pump scheduling in water supply. In: AISB international workshop on evolutionary computing. Springer, Berlin, Heidelberg, 1997. P. 227-235. Anais... https://doi. org/10.1007/BFb0027177

SNIS, 2017. Diagnóstico dos Serviços de Água e Esgotos - 2015. Disponivel em: <http://www.snis.gov.br/diagnostico-anual-agua-e-esgotos/diagnostico-ae-2015>. Acesso em: 27 abr. 2020.

SNIS, 2019. Diagnóstico dos Serviços de Água e Esgotos - 2018. Disponível em: <http://www.snis.gov.br/diagnostico-anual-agua-e-esgotos/diagnostico-dos-servicos-de-agua-e-esgotos-2018>. Acesso em: 28 abr. 2020.
TSUTIYA, M. T. Redução do custo de energia elétrica em sistemas de abastecimento de água. 2001.

USEPA, 2013. EPANET 2.0: Application for Modeling Drinking Water Distribution Systems. Disponível em: <http://www.epa. gov/nrmrl/wswrd/dw/epanet.html>. Acesso em: 26 abr. 2020.

VAN VELDHUIZEN, D. A. Multiobjective evolutionary algorithms: classifications, analyses, and new innovations. Air Force Inst Of Tech Wright-Pattersonafb Oh School Of Engineering, 1999.

VAN ZYL, J. E.; SAVIC, Dragan A.; WALTERS, G. A. Operational optimization of water distribution systems using a hybrid genetic algorithm. Journal of water resources planning and management, v. 130, n. 2, p. 160-170, 2004. https://doi.org/10.1061/ (ASCE)0733-9496(2004)130:2(160)

VIEIRA, B. S. et al. Optimizing drinking water distribution system operations. European Journal of Operational Research, v. 280 , n. 3, p. $1035-1050$, 2020. https://doi.org/10.1016/j. ejor.2019.07.060

VIEIRA, F.; RAMOS, H. M. Optimization of operational planning for wind/hydro hybrid water supply systems. Renewable Energy, v. 34, n. 3, p. 928-936, 2009. https://doi.org/10.1016/j.renene.2008.05.031

WALSKI, T. M. et al. Battle of the network models: Epilogue. Journal of Water Resources Planning and Management, American Society of Civil Engineers, v. 113, n. 2, p. 191-203, 1987. https:// doi.org/10.1061/(ASCE)0733-9496(1987)113:2(191)

WANG, J.; CHANG, T.; CHEN, J. An enhanced genetic algorithm for bi-objective pump scheduling in water supply. Expert Systems with Applications, v. 36, n. 7, p. 10249-10258, 2009. https://doi. org/10.1016/j.eswa.2009.01.054

YU, G.; POWELL, R. S.; STERLING, M. J. H. Optimized pump scheduling in water distribution systems. Journal of optimization theory and applications, v. 83, n. 3, p. 463-488, 1994. https://doi. org/10.1007/BF02207638

ZESSLER, U.; SHAMIR, Uri. Optimal operation of water distribution systems. Journal of water resources planning and management, v. 115, n. 6, p. 735-752, 1989. https://doi.org/10.1061/ (ASCE)0733-9496(1989)115:6(735) 